\title{
Noncoherent Correlation-Based Communication Systems Choosing Different Chaotic Maps
}

\author{
Shintaro ARAI ${ }^{\dagger}$ and Yoshifumi NISHIO ${ }^{\dagger}$ \\ $\dagger$ Department of Electrical and Electronic Engineering, Tokushima University \\ 2-1 Minami-Josanjima, Tokushima, 770-8506 JAPAN \\ Telephone: +81-88-656-7470, Fax: +81-88-656-7471 \\ Email: \{arai, nishio\} @ee.tokushima-u.ac.jp
}

\begin{abstract}
This paper proposes a new noncoherent detection system improved based on the differential chaos shift keying (DCSK) and the correlation delay shift keying (CDSK). In this scheme, a transmitter changes chaotic maps for generating a chaotic sequence efficiently depending on an initial value. Also, the proposed method can choose the chaotic map by a very simple algorithm. In order to investigate the proposed method, we carry out computer simulations and observe the performance.
\end{abstract}

\section{INTRODUCTION}

Recently, chaos-based digital communication systems are studied actively [1]- [5]. Especially, it is attracted to develop noncoherent detection systems which do not need to recover the basis signals (unmodulated carries) at the receiver. The differential chaos shift keying (DCSK) [1] and the optimal receiver [2] are well known as a typical noncoherent system. In addition, the correlation delay shift keying (CDSK) [3] similar to the DCSK scheme is also regarded.

In our previous research, we proposed a transmitter changing a chaotic sequence depending on an initial value for a suboptimal receiver [6], where the suboptimal receiver has a performance similar to the optimal receiver, and this algorithm is simpler than the optimal receiver. For the simulation results, it was observed that its performance was better than that of the conventional transmitter for the suboptimal receiver. From this result, we expected that the performances of the noncoherent detection systems also depend on chaotic maps used.

In this paper, we propose a new noncoherent detection system improved based on correlation-based noncoherent detection schemes. Specifically, the performance of the noncoherent communication system is improved by changing a chaotic map efficiently depending on an initial value in the transmitter. After describing the motivation and the system of the proposed method, we evaluate its performance by the computer simulation.

\section{SySTEM OVERVIEW}

In this research, we use the differential chaos shift keying (DCSK) [1] and the correlation delay shift keying (CDSK) [3] as the correlation-based noncoherent detection system. Each operation is described below.

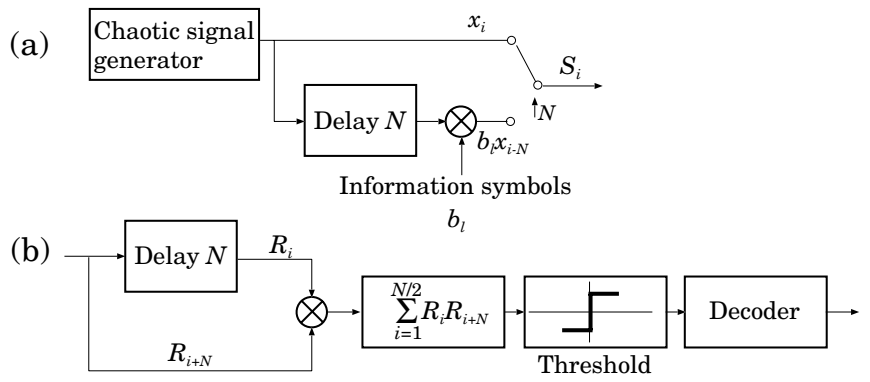

Fig. 1. DCSK operation. (a) transmitter. (b) receiver.

\section{A. $D C S K$}

Figure 1 shows the block diagram of a DCSK transmitter (a) and receiver (b). In this scheme, the transmitter outputs a chaotic sequence $x_{i}$ followed by the same sequence multiplied by the information symbol $b_{l}( \pm 1)$. In order to transmit 1-bit information, $N$ chaotic signals are generated, where $N$ is the chaotic sequence length. Therefore, the transmitted signal is given by

$$
S_{i}= \begin{cases}x_{i} & (1 \leq i \leq N) \\ b_{l} x_{i-N} & (N+1 \leq i \leq 2 N)\end{cases}
$$

Also, the transmitted signal can be written as $\mathbf{S}=$ $\left(\begin{array}{llll}S_{1} & S_{2} & \cdots & S_{2 N}\end{array}\right)$ by vector. Since the noise $\mathbf{n}=$ $\left(\begin{array}{llll}n_{1} & n_{2} & \cdots & n_{2 N}\end{array}\right)$ is added to the transmitted signal by the channel, the received signal can be written as $\mathbf{R}=$ $\left(\begin{array}{llll}R_{1} & R_{2} & \cdots & R_{2 N}\end{array}\right)=\mathbf{S}+\mathbf{n}$.

On the receiving side, it is evaluated by the correlation of 2 signals, which are obtained by dividing the received signals into two halves (length $N$ ). Thus, the output of the correlation can be written as

$$
C_{1}=\sum_{i=1}^{N} R_{i} R_{i+N}
$$

The decoded symbol is decided as " +1 " or " -1 " depending on $C_{1}$ being larger or smaller than 0 .

In order to generate the transmitted signal, the DCSK transmitter needs to switch correctly by the chaotic sequence length $N$. Therefore, the sophisticated switch is required, and it is regarded as the important issue to design DCSK. 
(a)

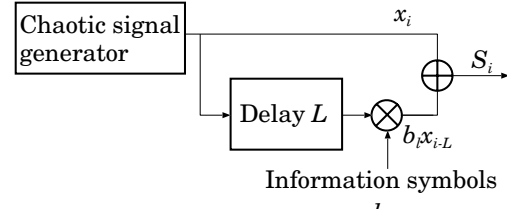

(b)

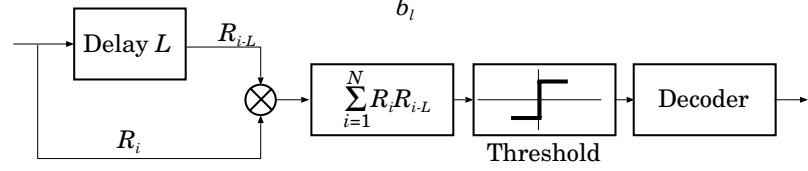

Fig. 2. CDSK operation. (a) transmitter. (b) receiver.

\section{B. $C D S K$}

Figure 2 shows the block diagram of a CDSK transmitter (a) and receiver (b). In this scheme, the transmitted signal is the sum of the chaotic sequence $x_{i}$ and its delayed sequence $x_{i-L}$ multiplied by the information symbol $b_{l}( \pm 1)$, where $L$ is the delay. Thus, the transmitted signal is given by

$$
S_{i}=x_{i}+b_{l} x_{i-L} .
$$

Since the sequence length is $N$ as with DCSK, we can also write the transmitted signal with the vector. The CDSK receiver is similar to the DCSK receiver. Therefore, the receiver can detect the information by multiplying the received signal and its delayed signal, as shown in Eq. (4).

$$
C_{2}=\sum_{i=1}^{N} R_{i} R_{i-L}
$$

As in the case of DCSK, the decoded symbol is decided as " +1 " or " -1 " depending on $C_{2}$ being larger or smaller than 0 .

As compared with DCSK, the CDSK transmitter does not need the switch to generate the transmitted signal. In other words, it means that operation of the transmitter can be performed continuously. Consequently, we can simplify and design the transmitter.

\section{Proposed Method}

\section{A. Motivation of Proposed Method}

Before describing the proposed method, we explain the motivation to use different chaotic maps.

As described in the introduction, it was observed that the effectiveness of the noncoherent detection system which changes the chaotic sequence length depends on the initial value. From this result, we expected that the performances of the noncoherent detection systems also depends on chaotic maps used. In order to investigate this expectation, we carried out the following computer simulation.

On the transmitting side, the interval of the initial value $[-1,1]$ is divided into 128 sections. We choose one section and selected $10^{4}$ initial points from this section at random. By using the chaotic sequences starting from these initial points, we transmit $10^{4}$ bits of information. In this study, we used the skew tent map, the skewed Bernoulli shift map and their reversal maps as chaotic maps, as shown in Fig. 3. In addition, the chaotic sequence length $N$ is 8 . Also, the delay $L$ of CDSK is 3 .

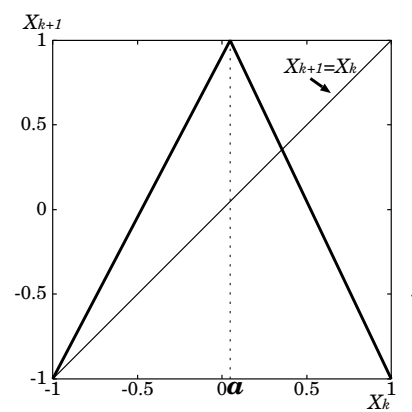

(a) Skew tent map.

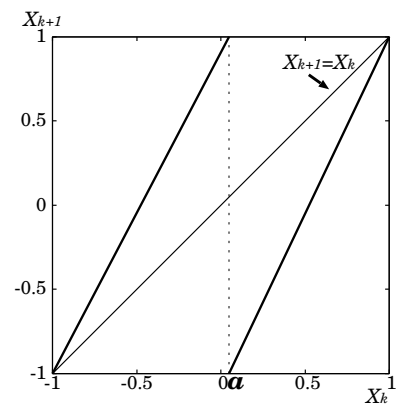

(c) Skewed Bernoulli shift map.

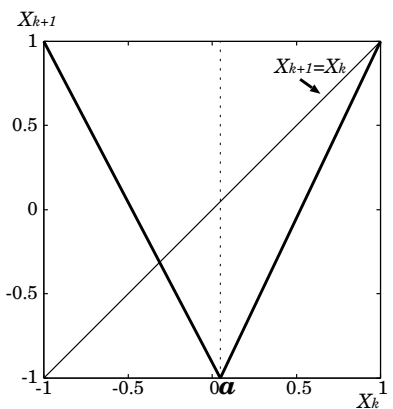

(b) Skew tent map (reversal).

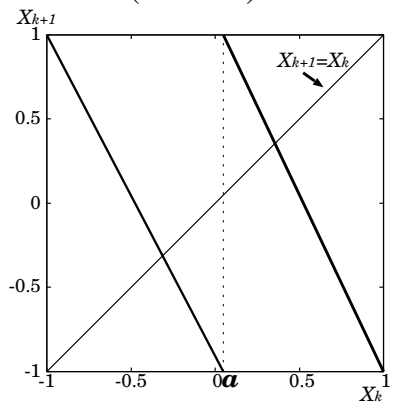

(d) Skewed Bernoulli shift map (reversal).
Fig. 3. Chaotic maps $(a=0.05)$.

Figures 4 and 5 show the simulation results of DCSK and CDSK when $E_{b} / N_{0}$ is $10 \mathrm{~dB}$, where the horizontal axes and the vertical axes are the section and the bit error rate (BER), respectively. From this figure, we can find that the BER strongly depends on the selected section. For example, in the case of DCSK, we can see that the BER of the reversal skew tent map is better than others for the 25th section, as shown in Fig. 4(b). However, in the 35th section, the skew tent map is better than others. Similarly, it can be also observed that each BER of chaotic maps changes for every section in the case of CDSK, as shown in Fig. 5(b). Furthermore, we can find that the better chaotic map of each section is different for DCSK and CDSK. These results mean that the performance of DCSK and CDSK is changed depending on the initial value of the chaotic sequence. In other words, it can be said that the performance of the noncoherent detection system is changed depending on the chaotic map.

From this result, we have devised new DCSK and CDSK transmitters choosing the chaotic map depending on the initial value of the chaotic sequence.

\section{B. Operation of Proposed Method}

Figures 6(a) and (b) show the block diagram of the proposed DCSK and CDSK transmitters, respectively. These transmitters are constructed by attaching two blocks to the conven- 


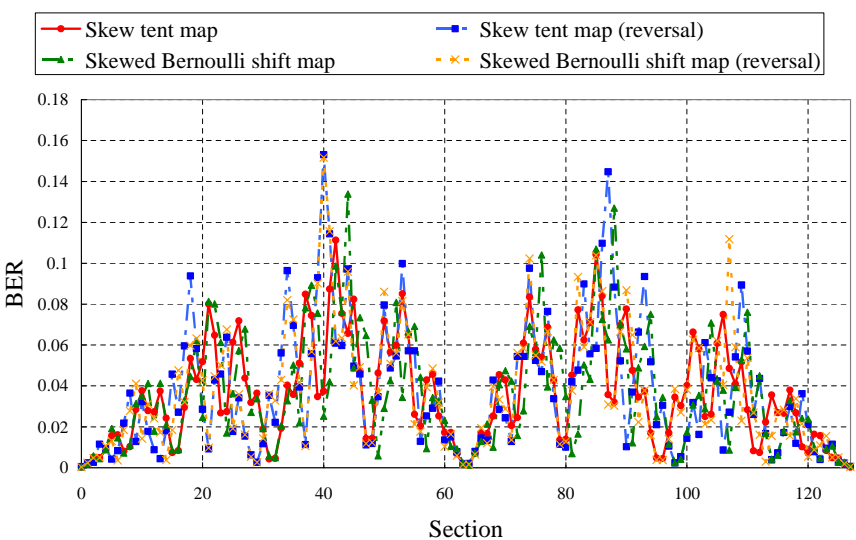

(a) Section $(0 \sim 127)$ versus BER $\left(E_{b} / N_{0}=10 \mathrm{~dB}\right)$.

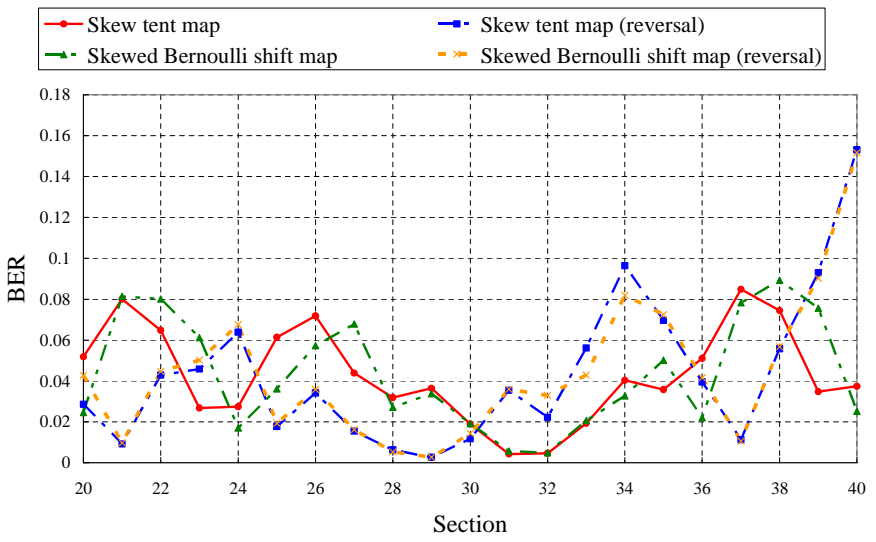

(b) Section $(20 \sim 40)$ versus BER $\left(E_{b} / N_{0}=10 \mathrm{~dB}\right)$.

Fig. 4. Basis of proposed method (DCSK).

tional transmitter. Selection of the chaotic map and generation of the transmitted signal are operated in the following order.

First, one section is determined by the initial value of the chaotic sequence in the 1st block. Next, a better chaotic map is chosen for the section in the 2nd block. Here, in order to choose the better chaotic map, we use the result obtained by the previous simulation in Figs. 4 and 5. Finally, the chaotic sequence is generated using the better chaotic map in the 3rd block. Also, the operation after choosing is the same as the conventional operation, namely, the transmitted signal can be similarly written with Eqs. (1) and (3). Moreover, the receiver of the conventional DCSK and CDSK can be also used for the detection of the information. By using the proposed method, the chaotic map can be changed efficiently and the transmission capability can be improved.

\section{Simulation Results AND Discussion}

In this section, we study the performance of the proposed method by computer simulations. The simulation conditions are as follows. In the transmitting side, the chaotic map is chosen from 4 types, the skew tent map, the skewed Bernoulli shift map and their reversal maps. Also, the chaotic sequence length $N$ is 8 or 16 , and the interval of the initial value, i.e., the section is 128 or 1024 . In CDSK, the delay $L$ is 3 . In the

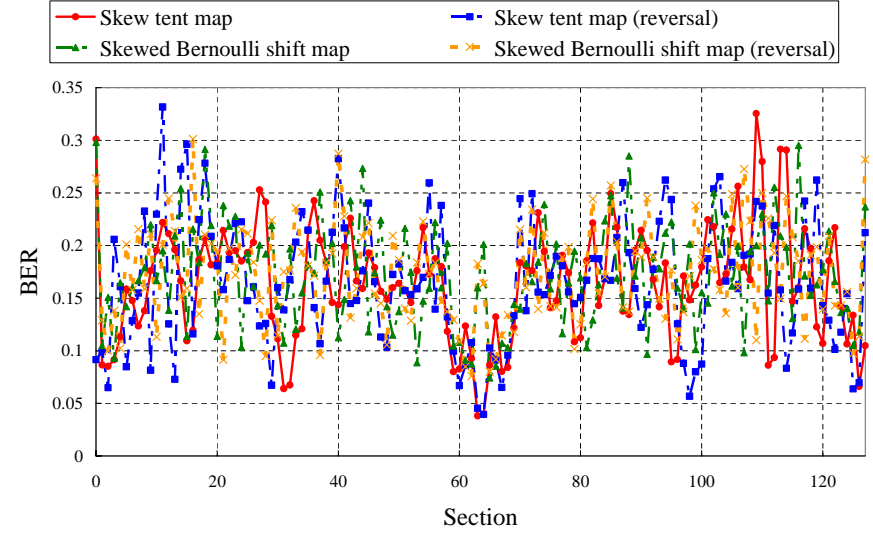

(a) Section $(0 \sim 127)$ versus BER $\left(E_{b} / N_{0}=10 \mathrm{~dB}\right)$.

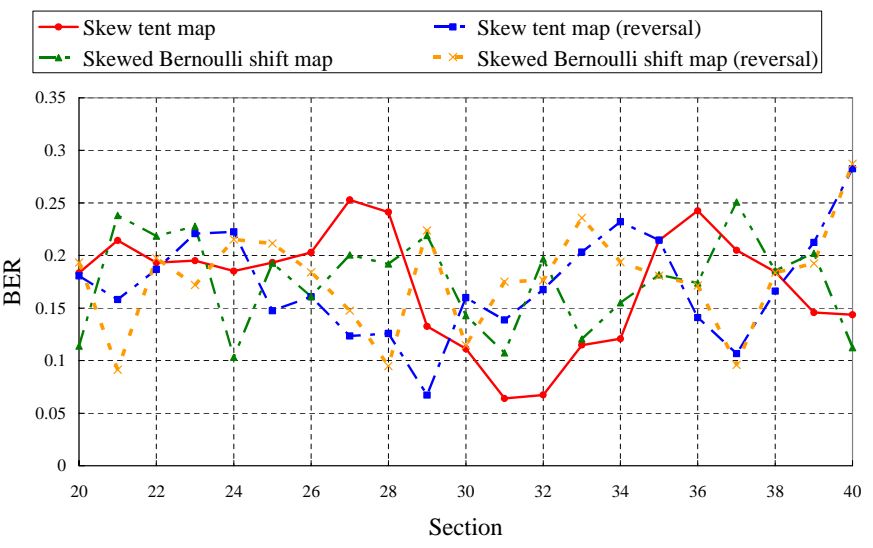

(b) Section $(20 \sim 40)$ versus BER $\left(E_{b} / N_{0}=10 \mathrm{~dB}\right)$.

Fig. 5. Basis of proposed method (CDSK).

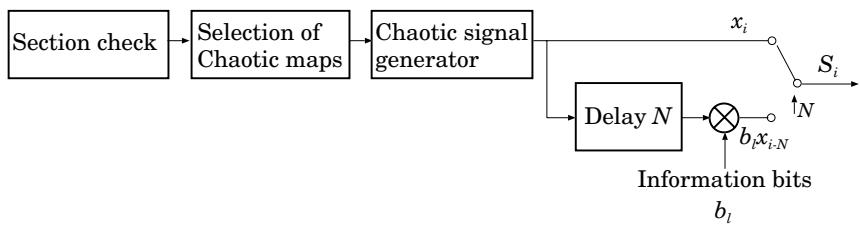

(a) Proposed DCSK transmitter.

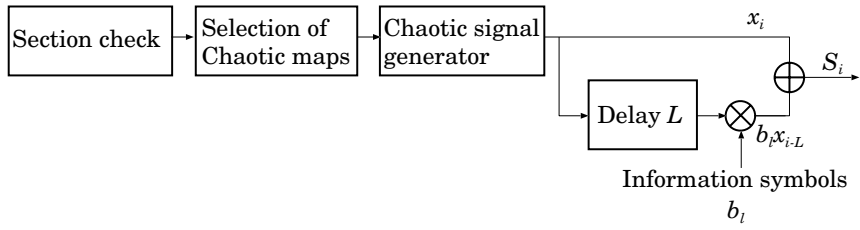

(b) Proposed CDSK transmitter.

Fig. 6. Proposed method.

channel, noise is assumed to be only AWGN. Hence, the noise at the transmitter and the receiver are not considered. On the receiving side, the same receiver as the conventional DCSK and CDSK is used. Based on these conditions, the system performance is evaluated by plotting the BER against $E_{b} / N_{0}$ when $10^{4}$ bits of information are transmitted.

Figure 7 shows the simulation results of the proposed method. In order to compare the performance of the proposed 
method, the performance of the conventional DCSK and CDSK is shown together in Fig. 7(a) and (b), respectively. In addition, Fig. 7(b) shows the comparison of performance for different values of the sections. From the result, it can be observed that the performance of these proposed methods is better than the both conventional system. In particular, the performance of the proposed CDSK improves significantly. However, as for the proposed DCSK method, the performance improve slightly. This reason is considered that transmitted energy has influenced. Table I shows the average transmitted energy per 1 chip of the chaotic sequence length. From this table, we can find that the energy per 1 chip of the proposed method is increasing. In the case of the DCSK, since the transmitter outputs the double sequence of the chaotic sequence length $N$, the overall transmitted energy has increased. On the other hand, since the transmitted signal of the CDSK becomes the length with the sequence $N$ added the Delay $L$, the sequence length of the CDSK is shorter than that of the DCSK. Namely, we consider that it influenced the result that the noise energy of the proposed DCSK became large than that of the conventional DCSK. However, although transmitted energy increased, the performance of the proposed method improved. Therefore, if the balance of transmitting energy and $\mathrm{BER}$ is regarded, we expect that the performance may improve further.

TABLE I

AVERAGE TRANSMITTED ENERGY PER 1 CHIP OF CHAOTIC SEQUENCE $\left(N=8, L=3\right.$, SECTION $\left.=128, E_{b} / N_{0}=10[\mathrm{DB}]\right)$.

\begin{tabular}{c|c|c}
\hline & Conventional method & Proposed method \\
\hline \hline DCSK & 0.333 & 0.392 \\
CDSK & 0.454 & 0.518 \\
\hline
\end{tabular}

Moreover, we can also confirm that the performance with many sections is better than that with few sections, as shown in Fig 7(b). As a reason, we assume that it is because the selection accuracy of the chaotic map improved by increasing the section. Thus, we consider that it is possible to improve the performance by narrowing the interval of this initial value in the case where the chaotic sequence length increases.

In general, increasing the chaotic sequence length of DCSK is not necessary to improve the performance. Also, the performance of CDSK changes significantly depending on the chaotic sequence length or the delay. However, we expect that these issues are solved by using the proposed method. Therefore, it can be said that choosing the chaotic map depending on the initial value is effective.

\section{CONCLUSION}

In this paper, we have proposed the new DCSK and CDSK transmitter changing chaotic maps depending on the initial value. We also evaluated the BER performance of the proposed method by comparing with the conventional DCSK and CDSK. As a result, it was observed that the performance of the proposed method is better than that of the conventional system, namely, we obtained the effectivity of the proposed method.

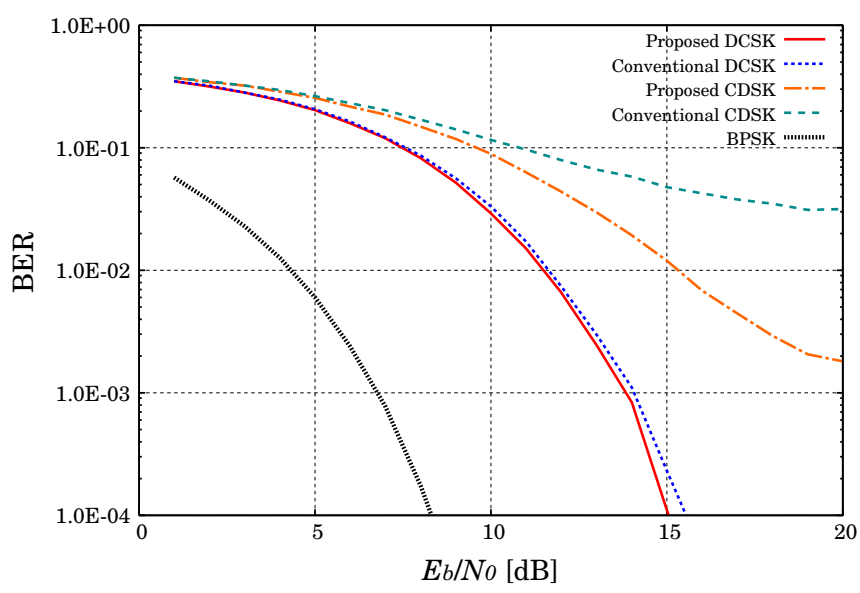

(a) $N=8$.

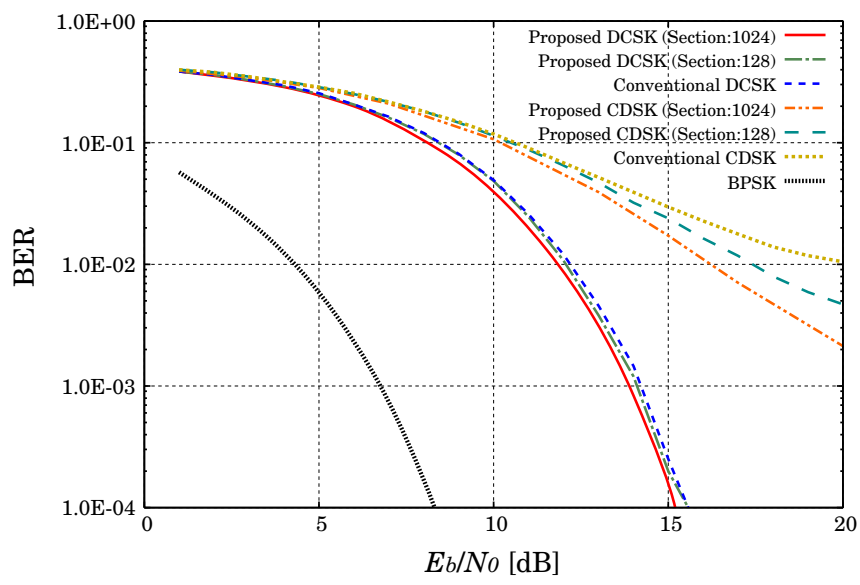

(b) $N=16$.

Fig. 7. Simulation results.

In this study, we carried out this simulation using the small chaotic sequence length. Thus, investigating the performance of the proposed method which increased the chaotic sequence length is our future work.

\section{REFERENCES}

[1] G. Kolumbán, B. Vizvári, W. Schwarz, and A. Abel, "Differential chaos shift keying: A robust coding for chaos communication,' Proc. NDES'96, pp. 87-92, Jun. 1996.

[2] M. Hasler and T. Schimming, "Chaos communication over noisy channels," Int . Journal of Bifurcation and Chaos, vol. 10, no. 4, pp. 719-736, Apr. 2000.

[3] M. Sushchik, L. S. Tsimring and A. R. Volkovskii, "Performance Analysis of Correlation-Based Communication Schemes Utilizing Chaos," IEEE Trans. Circuits and Systems Part I, vol. 47, no. 12, pp. 1684-1691, Dec. 2000.

[4] F. C. M. Lau and C. K. Tse, Chaos-Based Digital Communication Systems, Springer, 2003.

[5] W. M. Tam, F. C. M. Lau and C. K. Tse, "Generalized correlation-delayShift-Keying Scheme for Noncoherent Chaos-Based Communication Systems," IEEE Trans. Circuits and Systems Part I, vol. 53, no. 3, pp. 712 721, Mar. 2006

[6] S. Arai and Y. Nishio, "Variable Sequence Length Transmitter for Noncoherent Chaos Shift Keying," RISP Journal of Signal Processing, vol. 10, no. 4, pp. 255-258, Jul. 2006. 